\title{
Submillimeter spectroscopy and astronomical searches of vinyl mercaptan, $\mathrm{C}_{2} \mathrm{H}_{3} \mathrm{SH}^{\star}$
}

\author{
M.-A. Martin-Drumel ${ }^{1,2, \star \star}$, K. L. K. Lee ${ }^{2}$, A. Belloche ${ }^{3}$, O. Zingsheim ${ }^{1}$, S. Thorwirth ${ }^{1}$, H. S. P. Müller ${ }^{1}$, F. Lewen ${ }^{1}$, \\ R. T. Garrod ${ }^{4}$, K. M. Menten ${ }^{3}$, M. C. McCarthy ${ }^{2}$, and S. Schlemmer ${ }^{1}$
}

\author{
1 I. Physikalisches Institut, Universität zu Köln, Zülpicher Str. 77, 50937 Köln, Germany \\ e-mail: marie-aline.martin@u-psud.fr \\ 2 Harvard-Smithsonian Center for Astrophysics, 60 Garden Street, Cambridge, MA 02138, USA \\ 3 Max-Planck-Institut für Radioastronomie, Auf dem Hügel 69, 53121 Bonn, Germany \\ ${ }^{4}$ Departments of Astronomy \& Chemistry, University of Virginia, McCormick Road, PO Box 400319, Charlottesville, \\ VA 22904-4319, USA
}

Received 8 January 2019 / Accepted 13 February 2019

\begin{abstract}
Context. New laboratory investigations of the rotational spectrum of postulated astronomical species are essential to support the assignment and analysis of current astronomical surveys. In particular, considerable interest surrounds sulfur analogs of oxygencontaining interstellar molecules and their isomers.

Aims. To enable reliable interstellar searches of vinyl mercaptan, the sulfur-containing analog to the astronomical species vinyl alcohol, we investigated its pure rotational spectrum at millimeter wavelengths.

Methods. We extended the pure rotational investigation of the two isomers syn and anti vinyl mercaptan to the millimeter domain using a frequency-multiplication spectrometer. The species were produced by a radiofrequency discharge in 1,2-ethanedithiol. Additional transitions were remeasured in the centimeter band using Fourier-transform microwave spectroscopy to better determine rest frequencies of transitions with low- $J$ and low- $K_{\mathrm{a}}$ values. Experimental investigations were supported by quantum chemical calculations on the energetics of both the $\left[\mathrm{C}_{2}, \mathrm{H}_{4}, \mathrm{~S}\right]$ and $\left[\mathrm{C}_{2}, \mathrm{H}_{4}, \mathrm{O}\right]$ isomeric families. Interstellar searches for both syn and anti vinyl mercaptan as well as vinyl alcohol were performed in the EMoCA spectral line survey carried out toward Sgr B2(N2) with ALMA. Results. Highly accurate experimental frequencies (to better than $100 \mathrm{kHz}$ accuracy) for both syn and anti isomers of vinyl mercaptan are measured up to $250 \mathrm{GHz}$; these deviate considerably from predictions based on extrapolation of previous microwave measurements. Reliable frequency predictions of the astronomically most interesting millimeter-wave lines for these two species can now be derived from the best-fit spectroscopic constants. From the energetic investigations, the four lowest singlet isomers of the $\left[\mathrm{C}_{2}, \mathrm{H}_{4}, \mathrm{~S}\right]$ family are calculated to be nearly isoenergetic, which makes this family a fairly unique test bed for assessing possible reaction pathways. Upper limits for the column density of syn and anti vinyl mercaptan are derived toward the extremely molecule-rich star-forming region Sgr B2(N2) enabling comparison with selected complex organic molecules.
\end{abstract}

Key words. astrochemistry - molecular data - method: laboratory: molecular - ISM: individual objects: Sagittarius B2 (N) ISM: molecules - surveys

\section{Introduction}

From the diatomic $\mathrm{SH}^{+}$and $\mathrm{SH}$ radicals (Menten et al. 2011; Neufeld et al. 2012) to the large complex organic molecule ethyl mercaptan (ethanethiol, $\mathrm{C}_{2} \mathrm{H}_{5} \mathrm{SH}$; Kolesniková et al. 2014), nearly 20 sulfur-bearing species have been observed in the interstellar medium (ISM) and in the circumstellar envelopes of evolved stars. This total is substantial, representing roughly $10 \%$ of all the known interstellar or circumstellar compounds. While the abundance of S-containing species in the diffuse ISM is consistent with the estimated cosmic abundances of sulfur (Savage \& Sembach 1996) and is well reproduced by current astrophysical models (Neufeld et al. 2015), the same set of compounds only accounts for $\sim 0.1 \%$ of the expected sulfur abun-

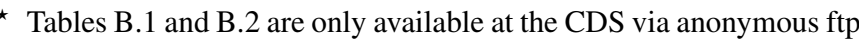
to cdsarc.u-strasbg. fr (130.79.128.5) or via http://cdsarc. u-strasbg.fr/viz-bin/qcat?]/A+A/623/A167

$\star \star$ Present address: Institut des Sciences Moléculaires d'Orsay (ISMO), CNRS, Univ. Paris-Sud, Université Paris-Saclay, 91405 Orsay, France.
}

dance in the cold, dense clouds and circumstellar regions around young stellar objects (Joseph et al. 1986; Tieftrunk et al. 1994). This remarkable disparity has given rise to a simple, but currently unanswered, question: Where is the missing sulfur? Several potential reservoirs have been investigated, including atomic sulfur (Anderson et al. 2013), S-bearing species trapped on icy dust grains (Jimenez-Escobar \& Munoz Caro 2011), and S-containing molecules in the gas phase (Martin-Domenech et al. 2016; Cazzoli et al. 2016), along with other possibilities. From studies of shocked gas, however, atomic sulfur can only account for at most $5-10 \%$ of the total sulfur cosmic abundance (Anderson et al. 2013), implying that additional sulfur sinks must exist. Many studies propose that the depletion of sulfur reflects accretion onto dust grains, where it could be sequestered in various forms as inorganic and organic molecules (Scappini et al. 2003), or atomic and polymeric $S_{n}$ chains and rings (Wakelam et al. 2004). So far, however, only two sulfur molecules, OCS and $\mathrm{SO}_{2}$, have been detected on grains, both in too low abundance for either species to be a major reservoir of sulfur (Palumbo et al. 1995; Boogert et al. 1997). Since $\mathrm{H}_{2} \mathrm{~S}$ 
is the most abundant sulfur-bearing molecule in cometary ices (Bockelee-Morvan et al. 2000), its presence on ice grains has also received some attention, but the rather low upper limit on its abundance also appears to rule out this species as a major sink for sulfur (Smith 1991; Jimenez-Escobar \& Munoz Caro 2011). Finally, photoprocessing of sulfur-containing ice products could yield gaseous S-bearing species in the dense ISM in molecular forms that are yet to be identified (Jimenez-Escobar \& Munoz Caro 2011). Consequently, the identification of missing interstellar sulfur carriers requires the detection of new gaseous sulfur containing compounds, which in turn requires laboratory investigations of their rotational spectra. Transient species are particularly appealing candidates, but are often challenging to characterize in the laboratory compared to their stable, often commercially available, counterparts, especially in the millimeter- and submillimeter-wave region in which highaltitude, high-sensitivity radio facilities such as the Atacama Large Millimeter/submillimeter Array (ALMA) operate.

While Kolesniková et al. (2014) reported the detection of ethyl mercaptan in Orion, its presence there is not without controversy (Müller et al. 2016). Regardless, experimental data on related molecules that might also be present in the ISM and participate in the same chemical network, for instance unsaturated variants, may prove helpful in resolving this controversy. The isomeric family with the elemental formula $\left[\mathrm{C}_{2}, \mathrm{H}_{4}, \mathrm{~S}\right]-$ thioacetaldehyde $\left(\mathrm{CH}_{3} \mathrm{CHS}\right)$, thiirane $\left(c-\mathrm{C}_{2} \mathrm{H}_{4} \mathrm{~S}\right)$, and vinyl mercaptan $\left(\mathrm{CH}_{2} \mathrm{CHSH}\right)-$ are of particular interest as all three isovalent oxygen counterparts (acetaldehyde, oxirane, and vinyl alcohol) have been detected in the ISM (Gottlieb 1973; Dickens et al. 1997; Turner \& Apponi 2001). Structural isomers are an invaluable tool to probe the chemical dynamics of astrophysical environments. While a minimum energy principle was once proposed to assert molecular abundance in space (Lattelais et al. 2009), the general consensus is that chemical kinetics, rather than thermodynamic stability, is driving the formation of molecules in astronomical sources (Loomis et al. 2015). Interestingly, relatively little is known about isomers of relatively large astrophysical species (five atoms and more); this is in part because of the difficulties involved in both producing and detecting these species in the laboratory, and the $\left[\mathrm{C}_{2}, \mathrm{H}_{4}, \mathrm{~S}\right]$ family is no exception. While the pure rotational spectrum of each of these three singlet isomers has been investigated in the microwave domain (Kroto et al. 1974; Cunningham et al. 1951; Tanimoto \& Saito 1977), high-resolution measurements at millimeter and shorter wavelengths have only been published for thiirane (Hirao et al. 2001; Bane et al. 2012), which is a likely reflection of the stability of this compound in the laboratory. In contrast, thioacetaldehyde polymerizes rapidly, with a lifetime on the order of ten seconds (Kroto et al. 1974), and consequently it must, as with vinyl mercaptan, be produced in situ for spectroscopic studies. To confidently detect these two transient species in the ISM, laboratory measurements at higher frequency are essential, particularly in spectral bands in which ALMA operates.

In this study we measured the pure rotational spectrum of the syn and anti isomers of vinyl mercaptan in their ground vibrational state up to $250 \mathrm{GHz}$. From the newly recorded transitions, we derived highly accurate rotational and centrifugal distortion constants for both species, ensuring that astronomical searches in the millimeter domain can now be undertaken with confidence. Using the new experimental data, we carried out searches on the spectral line survey EMoCA (Exploring Molecular Complexity with ALMA) performed toward the region of high-mass star formation Sagittarius (Sgr) B2(N) (Belloche et al. 2016). As part of this work, we also investigated the energetics of the $\left[\mathrm{C}_{2}, \mathrm{H}_{4}, \mathrm{~S}\right]$
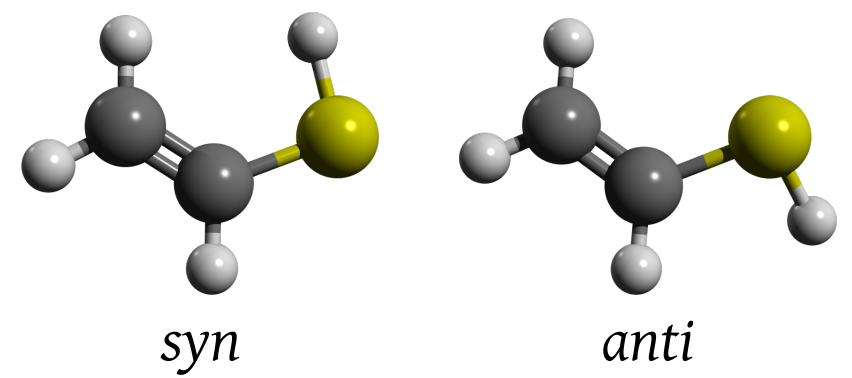

Fig. 1. Molecular representation of planar syn and quasi-planar anti vinyl mercaptan.

isomeric family and its isovalent oxygen counterparts via highlevel quantum chemical calculations.

\section{Methods}

\subsection{Spectroscopic background}

Vinyl mercaptan exists in the gas phase in two distinct rotameric forms, the planar syn and quasi-planar anti isomers (Fig. 1); the former is only slightly more stable than the latter by about $0.6 \mathrm{~kJ} \mathrm{~mol}^{-1}$ (Almond et al. 1983, 1985; Plant et al. 1992), which yields a syn/anti ratio at room temperature of about 1.2. It is worth noting that while the equilibrium structure of the anti isomer is non-planar, with a calculated $\mathrm{SH}$ torsional angle of about $130-150^{\circ}$, the barrier to linearity $\left(0.14 \mathrm{~kJ} \mathrm{~mol}^{-1}\right)$ is small compared to the zero-point vibration, which results in a vibrationally averaged planar structure (Tanimoto \& Macdonald 1979; Almond et al. 1985). As a consequence, both isomers can be considered as belonging to the $C_{\mathrm{s}}$ point group symmetry (Almond et al. 1983).

Experimental studies on vinyl mercaptan are limited. Photoelectron (Chin et al. 1994) and low-resolution vibrational spectra (Almond et al. 1977, 1983) have been reported, while pure rotational spectra of the two isomers in the ground and several excited vibrational states have only been measured up to $50 \mathrm{GHz}$ (Almond et al. 1977; Tanimoto \& Saito 1977; Tanimoto et al. 1979; Tanimoto \& Macdonald 1979). Isotopic investigations of the microwave spectra of the SD isotopologs ${ }^{1}$ (Tanimoto \& Saito 1977; Tanimoto et al. 1979; Tanimoto \& Macdonald 1979), and extensive studies of deuterated variants of the syn isomer (Almond et al. 1985) that followed, enabled a determination of a partial geometry. Owing to the absence of experimental data for isotopic species involving substitution at any of the three heavy atoms, a complete structure has not been reported. Additionally, accurate dipole moments in the ground vibrational state are available from Stark measurements: $\mu_{\mathrm{a}}=0.813(1) \mathrm{D}$, $\mu_{\mathrm{b}}=0.376(4) \mathrm{D}, \mu_{\mathrm{tot}}=0.896(3) \mathrm{D}$ for the syn isomer; and $\mu_{\mathrm{a}}=0.425(10) \mathrm{D}, \mu_{\mathrm{b}}=1.033(10) \mathrm{D}, \mu_{\mathrm{tot}}=1.117(14) \mathrm{D}$ for the anti isomer (Tanimoto et al. 1979; Tanimoto \& Macdonald 1979).

\subsection{Computational details}

Quantum-chemical calculations of the $\left[\mathrm{C}_{2}, \mathrm{H}_{4}, \mathrm{~S}\right]$ and $\left[\mathrm{C}_{2}, \mathrm{H}_{4}, \mathrm{O}\right]$ isomeric families were performed at the coupled-cluster level with single, double, and perturbative triple excitations [CCSD(T)] (Raghavachari et al. 1989). All calculations were performed using the Coupled-Cluster techniques for

\footnotetext{
1 I.e., in which the single hydrogen atom bonded to the $S$ atom is replaced by a deuterium atom.
} 
Table 1. Ground state molecular parameters (in MHz) of syn and anti vinyl mercaptan derived in this study compared with previously published values (Tanimoto et al. 1979; Tanimoto \& Macdonald 1979) and those calculated theoretically (Calc.).

\begin{tabular}{|c|c|c|c|c|c|c|}
\hline & \multicolumn{3}{|c|}{ syn- $\mathrm{CH}_{2} \mathrm{CHSH}$} & \multicolumn{2}{|c|}{ anti- $\mathrm{CH}_{2} \mathrm{CHSH}$} & \multirow[b]{2}{*}{ Calc. $^{b}$} \\
\hline & This work $^{a}$ & Previous $^{a}$ & Calc. $^{b}$ & This work $^{a}$ & Previous $^{a}$ & \\
\hline$A_{\mathrm{e}}$ & $\ldots$ & $\ldots$ & 50304.4 & $\ldots$ & $\ldots$ & 49843.5 \\
\hline$\Delta A_{0}$ & $\cdots$ & $\cdots$ & 431.2 & $\cdots$ & $\cdots$ & 36.4 \\
\hline$B_{\mathrm{e}}$ & $\cdots$ & $\cdots$ & 5866.0 & $\cdots$ & $\cdots$ & 5912.5 \\
\hline$\Delta B_{0}$ & $\ldots$ & $\ldots$ & 38.1 & $\ldots$ & $\ldots$ & 31.9 \\
\hline$C_{\mathrm{e}}$ & $\ldots$ & $\ldots$ & 5253.4 & $\ldots$ & $\ldots$ & 5308.9 \\
\hline$\Delta C_{0}$ & $\ldots$ & $\ldots$ & 37.3 & $\ldots$ & $\ldots$ & 50.6 \\
\hline$A_{0}$ & $49816.0376(11)$ & $49815.28(6)$ & 49873.2 & $49423.5726(11)$ & $49422.75(5)$ & 49807.1 \\
\hline$B_{0}$ & $5835.708685(92)$ & $5835.716(14)$ & 5827.9 & $5897.21162(11)$ & $5897.215(9)$ & 5880.6 \\
\hline$C_{0}$ & $5222.075531(85)$ & $5222.081(11)$ & 5216.1 & $5279.43951(13)$ & $5279.436(9)$ & 5258.3 \\
\hline$D_{J} \times 10^{3}$ & $2.724203(89)$ & $2.85(17)$ & 2.716 & $3.09729(17)$ & $3.12(11)$ & 3.117 \\
\hline$D_{J K} \times 10^{3}$ & $-33.4874(12)$ & $-33.2(21)$ & -35.89 & $-37.6145(40)$ & $-38.5(17)$ & -40.02 \\
\hline$D_{K}$ & $0.79113(14)$ & $\ldots$ & 0.8100 & $0.80990(18)$ & $\ldots$ & 0.9496 \\
\hline$d_{1} \times 10^{3}$ & $-0.424456(88)$ & $-0.425(35)$ & -0.4239 & $-0.47324(19)$ & $-0.498(51)$ & -0.4143 \\
\hline$d_{2} \times 10^{3}$ & $-0.023739(73)$ & $\ldots$ & -0.0211 & $-0.03110(25)$ & $\ldots$ & -0.0411 \\
\hline$H_{K J} \times 10^{6}$ & $-3.1367(59)$ & $\cdots$ & $\ldots$ & $-4.356(31)$ & $\cdots$ & $\ldots$ \\
\hline \# of lines & 333 & 37 & $\cdots$ & 205 & 32 & $\cdots$ \\
\hline $\mathrm{RMS} / \mathrm{kHz}$ & 43 & $\cdots$ & $\cdots$ & 49 & $\cdots$ & $\cdots$ \\
\hline$\sigma^{c}$ & 0.95 & $\ldots$ & $\ldots$ & 1.03 & $\ldots$ & $\ldots$ \\
\hline
\end{tabular}

Notes. ${ }^{(a)}$ Numbers in parentheses are $1 \sigma$ uncertainties in units of the last digit. ${ }^{(b)} A_{\mathrm{e}}, B_{\mathrm{e}}$, and $C_{\mathrm{e}}$ rotational constants derived from structure calculated at the ae-CCSD $(\mathrm{T}) / \mathrm{cc}$-pwCVQZ level of theory. Zero-point vibrational corrections $\Delta A_{0}, \Delta B_{0}$, and $\Delta C_{0}$ and quartic centrifugal distortion constants were calculated at the fc-CCSD $(\mathrm{T}) / \mathrm{cc}-\mathrm{pV}(\mathrm{T}+d) \mathrm{Z}$ level. ${ }^{(c)}$ Reduced standard deviation (unitless).

Computational Chemistry (CFOUR) program package (Stanton et al. 2017; Harding et al. 2008a) in combination with Dunning's hierarchies of correlation consistent polarized valence and polarized core-valence sets (Dunning et al. 2001; Peterson \& Dunning 2002). The best estimate equilibrium structures have been calculated at the all-electron (ae) CCSD(T)/cc-pwCVQZ level of theory, which has previously been shown to yield equilibrium structures of very high quality for molecules harboring second-row elements (see, e.g., Coriani et al. 2005; Thorwirth \& Harding 2009; Thorwirth et al. 2015). Centrifugal distortion constants and zero-point vibrational corrections $\Delta B_{0}$ to the rotational constants were calculated in the frozen-core (fc) approximation at the fc-CCSD(T)/cc-pV(T+d)Z level and ground state rotational constants were obtained as $B_{0}=B_{\mathrm{e}}-\Delta B_{0}$. In this work, the equilibrium rotational constants $B_{\mathrm{e}}$ are calculated from the ae-CCSD(T)/cc-pwCVQZ structures. The calculated spectroscopic parameters for both syn and anti vinyl mercaptan are given in Table 1, and the elements of their Z-matrices are given in Appendix A.

The relative energetics of the $\left[\mathrm{C}_{2}, \mathrm{H}_{4}, \mathrm{O}\right]$ and $\left[\mathrm{C}_{2}, \mathrm{H}_{4}, \mathrm{~S}\right]$ isomers were calculated using a composite thermochemistry scheme similar to the High Accuracy Extrapolated Ab initio Thermochemistry (HEAT) approaches (see Bomble et al. 2006; Harding et al. 2008b). Using the CCSD(T)/cc-pwCVQZ geometries, a series of additive contributions are calculated: the extrapolated $\operatorname{CCSD}(\mathrm{T}) /$ aug-cc-pCVXZ $(X=\mathrm{D}, \mathrm{T}, \mathrm{Q})$ correlation energy; the correlation energy from perturbative quadruple excitations with fc-CCSDT(Q)/cc-pVDZ; the anharmonic zero-point contribution with $\operatorname{CCSD}(\mathrm{T}) / \mathrm{cc}-\mathrm{pV}(\mathrm{T}+d) \mathrm{Z}$; the diagonal Born-Oppenheimer correction with HF/aug-cc-pVTZ; and 1- and 2-electron scalar relativistic corrections with CCSD(T)/aug-cc-pCVTZ.

The relative stabilities, with $\mathrm{CH}_{3} \mathrm{CHO}$ and $\mathrm{CH}_{3} \mathrm{CHS}$ as the point of reference, are shown in Fig. 2. The energetic ordering

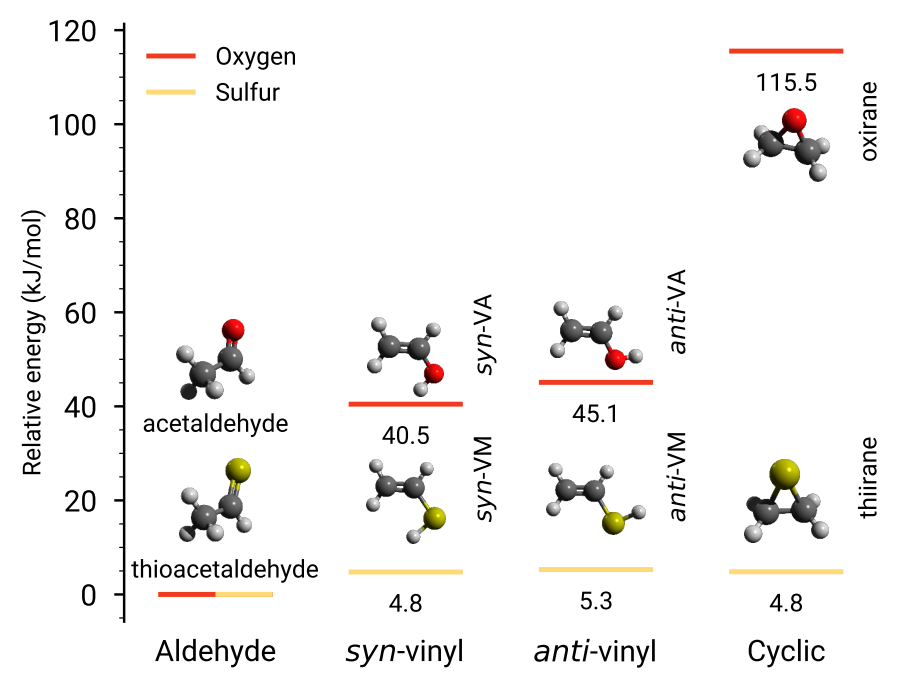

Fig. 2. Energetics of the $\left[\mathrm{C}_{2}, \mathrm{H}_{4}, X\right]$ species $(X=\mathrm{O}$ or $\mathrm{S})$, relative to the respective aldehydic forms, i.e., acetaldehyde and thioacetaldehyde, both set to $0 \mathrm{~kJ} \mathrm{~mol}^{-1}$.

of $\left[\mathrm{C}_{2}, \mathrm{H}_{4}, \mathrm{O}\right]$ isomers is in agreement with previous calculations by Lattelais et al. (2009) and Karton \& Talbi (2014): $\mathrm{CH}_{3} \mathrm{CHO}$ is the most stable isomer, followed by vinyl alcohol and oxirane. In particular, the energetics computed at the W2-F12 level by Karton \& Talbi (2014) for the syn isomer of vinyl alcohol are within $\sim 1 \mathrm{~kJ} \mathrm{~mol}^{-1}$ of the values presented in this work. The energy difference between the syn and anti isomers has been examined theoretically (Nobes et al. 1981) and experimentally; the value presented $\left(4.6 \mathrm{~kJ} \mathrm{~mol}^{-1}\right)$ is in excellent agreement with both the microwave $\left(4.5 \pm 0.6 \mathrm{~kJ} \mathrm{~mol}^{-1}\right.$, Rodler 1985) and the far-infrared semi-experimental determination $\left(4.0 \mathrm{~kJ} \mathrm{~mol}^{-1}\right.$, 


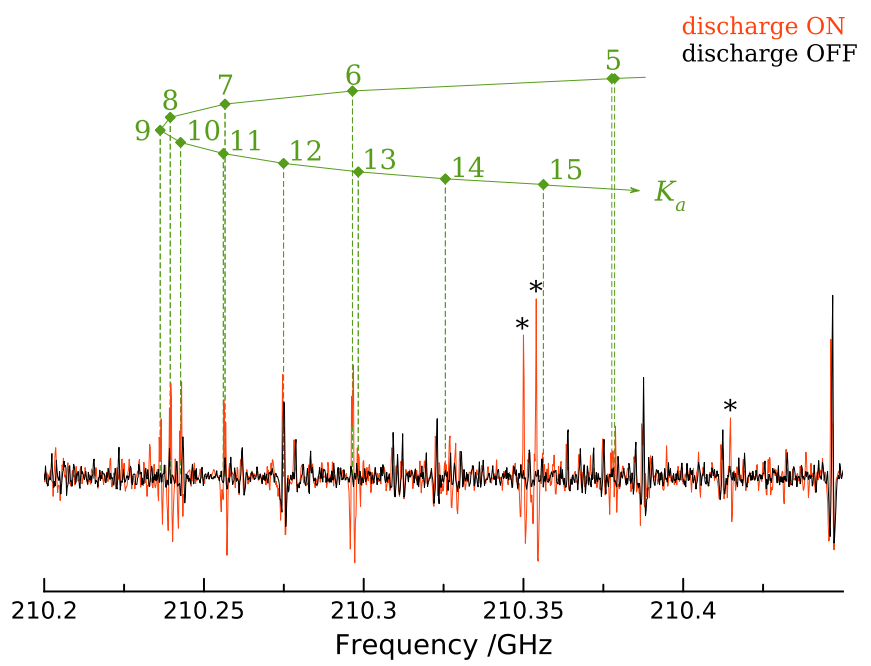

Fig. 3. Portion of the millimeter-wave spectrum recorded with RF discharge on (in red) and off (in black) showing the $K_{\mathrm{a}}$ series in the ${ }^{q} R(18)$ $\left(J^{\prime} \leftarrow J^{\prime \prime}=19 \leftarrow 18\right)$ branch of syn vinyl mercaptan. For the lowest $K_{\mathrm{a}}$ value visible on the plot, the asymmetry splitting $\left(K_{\mathrm{a}}+K_{\mathrm{c}}=J\right.$ or $J+1)$ is resolved. Transitions in black most likely arise from the precursor while transitions in red labeled with an asterisk remain unidentified (see text).

Bunn et al. 2017). The sulfur analogs $\left[\mathrm{C}_{2}, \mathrm{H}_{4}, \mathrm{~S}\right]$ on the other hand are near-isoenergetic at the current level of treatment. With the exception of $\mathrm{CH}_{3} \mathrm{CHS}$, the three remaining isomers are within $\sim 0.5 \mathrm{~kJ} \mathrm{~mol}^{-1}$ of one another, where anti-vinyl mercaptan is the highest in energy $\left(5.3 \mathrm{~kJ} \mathrm{~mol}^{-1}\right)$. Our ab initio energy difference between the vinyl mercaptan isomers $\left(0.5 \mathrm{~kJ} \mathrm{~mol}^{-1}\right)$ is in quantitative agreement with the $0.6 \mathrm{~kJ} \mathrm{~mol}^{-1}$ estimate by Almond et al. (1985).

\subsection{Millimeter-wave spectroscopy}

Millimeter-wave measurements on vinyl mercaptan were performed using the Cologne (sub)millimeter direct absorption spectrometer in the spectral regions $70-120 \mathrm{GHz}$ and $170-250 \mathrm{GHz}$. The radiation was produced by a commercial amplifier-multiplier chain (Virginia Diodes Inc.) and optimized by in-house electronics. To reduce $1 / f$ noise, frequency modulation was used with a $2 f$ demodulation, yielding line shapes close to a second-derivative for all recorded transitions. The $5 \mathrm{~m}$ long discharge cell, equipped with a roof-top mirror on one end, allows $10 \mathrm{~m}$ of absorption path length. A detailed description of the apparatus can be found in Martin-Drumel et al. (2015). Vinyl mercaptan was produced by a radio-frequency (RF) discharge in a continuous flow of 1,2-ethanedithiol $\left(\mathrm{HSCH}_{2} \mathrm{CH}_{2} \mathrm{SH}\right)$ at a pressure of about $10 \mu$ bar. It should be noted, however, that since the pressure was measured between the cell and pumping stage, a sizable pressure gradient may have been present in the cell. Similar to a previous study on OSSO (Martin-Drumel et al. 2015), the strongest signals of vinyl mercaptan were observed when the RF power was reduced to fairly low powers. However, to maintain stable discharge conditions in the cell, and thus to limit the noise induced by a "blinking" plasma, a discharge power of no less than $5 \mathrm{~W}$ was applied.

Upon ignition of the discharge, relatively strong transitions of both syn and anti isomers of vinyl mercaptan were observed, as illustrated in Fig. 3. To derive rest frequencies as accurate as possible, rotational transitions were measured individually using frequency steps of 10 and $20 \mathrm{kHz}$ in the $70-120 \mathrm{GHz}$ and $170-$

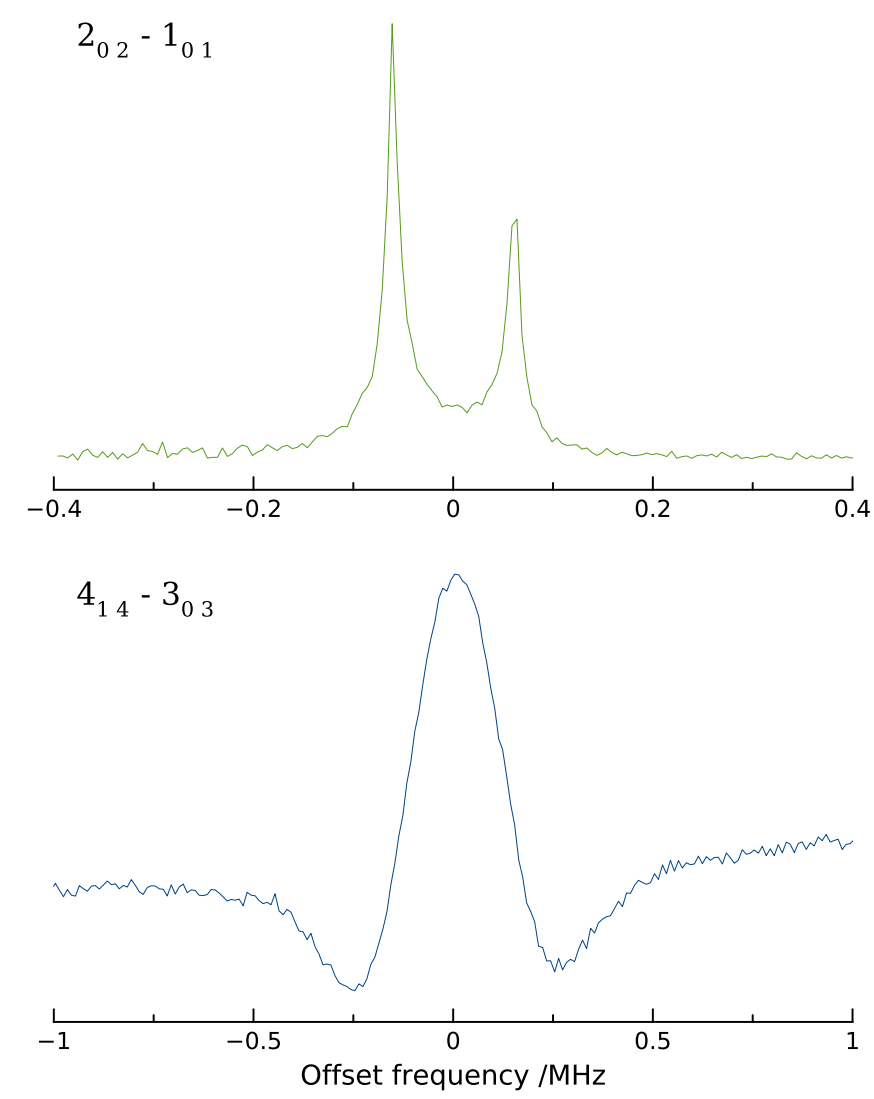

Fig. 4. Two transitions of anti vinyl mercaptan recorded in the microwave (top trace, in green) and millimeter-wave (bottom trace, in blue) domains. The frequency axis is given as an offset from the rest frequencies, 22346.6731 and $85467.985 \mathrm{MHz}$, respectively. The doublet structure of the microwave transition (top spectrum) is caused by Doppler splitting, which arises because of the coaxial arrangement of the cavity axis with respect to the axis along which the gas expands; the rest frequency is taken as the average of the two components. The second derivative line shape aspect of the millimeter transition (bottom spectrum) arises from $2 f$ demodulation of the modulated frequency.

$250 \mathrm{GHz}$ regions, respectively, and signal averaging was used to reach a signal-to-noise ratio of at least 10, and up to 50 in some cases (Fig. 4). The accuracy on each line frequency was derived from a fit to the line profile, accounting for the full width at half maximum (FWHM), frequency step, and signalto-noise ratio as described in Martin-Drumel et al. (2015). Using this approach, rest frequencies were derived with uncertainties as low as $10 \mathrm{kHz}$. Ultimately, nearly 300 and 150 new transitions (both $a$ - and $b$-type) of syn and anti isomers of vinyl mercaptan were recorded in the millimeter domain with quantum numbers up to $J^{\prime \prime}=22, K_{\mathrm{a}}^{\prime \prime}=17$ and $J^{\prime \prime}=19, K_{\mathrm{a}}^{\prime \prime}=14$, respectively. We note that, besides vinyl mercaptan, other species were produced under our experimental conditions (as indicated in Fig. 3). Although some of their transitions were observed with high signal-to-noise ratios, they could not be assigned to any known species using the Cologne Database for Molecular Spectroscopy (CDMS) and NASA Jet Propulsion Laboratory (JPL) catalogs (Endres et al. 2016; Pickett et al. 1998). The carriers of these lines may be vibrational satellites of vinyl mercaptan or its precursor, 1,2-ethanedithiol, or other species produced in the discharge. Since no wide spectral surveys were conducted, however, no further line assignment attempts were made at this juncture. 


\subsection{Centimeter-wave spectroscopy}

For each isomer of vinyl mercaptan, roughly ten transitions previously observed by Tanimoto et al. (1979) and Tanimoto \& Macdonald (1979) were remeasured between 10 and $35 \mathrm{GHz}$ using the Cambridge Fourier-transform microwave spectrometer, which was described elsewhere (McCarthy et al. 2000). In doing so, uncertainties on the rest frequencies of these lines were reduced by nearly two orders of magnitude, from $100 \mathrm{kHz}$ to $1 \mathrm{kHz}$. In this experiment, vinyl mercaptan was generated by a $1 \mathrm{kV}$ DC discharge in the throat of a small nozzle through a mixture of $\mathrm{C}_{2} \mathrm{H}_{2}$ and $\mathrm{H}_{2} \mathrm{~S}$ heavily diluted in neon and the resulting products were supersonically expanded into a large vacuum chamber. By the time the products reach the center of the FabryPerot cavity, where they are probed by the microwave radiation, the rotational temperature falls to $10 \mathrm{~K}$ or less. An example of a microwave transition of syn- $\mathrm{CH}_{2} \mathrm{CHSH}$ is presented in Fig. 4.

\subsection{Astronomical observations}

We searched for vinyl mercaptan in the EMoCA spectral line survey performed toward the Sgr B2(N) star-forming region with ALMA. We focused our analysis on the hot molecular core $\mathrm{Sgr} \mathrm{B} 2(\mathrm{~N} 2)$ at the position $\left(\alpha, \delta_{\mathrm{J} 2000}\right)=$ $\left(17^{\mathrm{h}} 47^{\mathrm{m}} 19.86^{\mathrm{s}},-28^{\circ} 22^{\prime} 13.4^{\prime \prime}\right)$. Details about the observations, data reduction, and method used to identify the detected lines and derive column densities can be found in Belloche et al. (2016). In short, the EMoCA survey covers the frequency range from $84.1 \mathrm{GHz}$ to $114.4 \mathrm{GHz}$ with a spectral resolution of $488.3 \mathrm{kHz}$ (1.7 to $\left.1.3 \mathrm{~km} \mathrm{~s}^{-1}\right)$. It was performed with five frequency tunings called $\mathrm{S} 1$ to $\mathrm{S} 5$. The median angular resolution is $1.6^{\prime \prime}$.

\section{Results and discussion}

\subsection{Rest frequencies and derived spectroscopic parameters}

The transitions recorded in this study are summarized in Appendix B. From these measurements, and previously published data (Tanimoto et al. 1979; Tanimoto \& Macdonald 1979), a fit to a Watson- $S$ Hamiltonian in the $\mathrm{I}^{r}$ representation was performed using the SPFIT/SPCAT suite of programs (Pickett 1991). The resulting best-fit spectroscopic constants are given in Table 1.

The large number of transitions recorded in this work coupled with the detection of lines that originate from much higher values of $J$ and $K_{\mathrm{a}}$ compared to the previous studies enable centrifugal distortion (CD) parameters up to sixth order to be precisely determined. In some cases, the precision of these CD parameters has been improved by as much as four orders of magnitude. To reproduce the measured transitions to their experimental accuracy, a total of nine parameters are required, including two quartic CD terms $\left(D_{K}\right.$ and $\left.d_{2}\right)$ and one sextic term $\left(H_{K J}\right)$ that were not determined previously. The much larger dataset used in the present investigation and the relatively small number of additional parameters needed point to the rapid convergence of the Hamiltonian model and the robustness of the fit. On the basis of the best-fit parameters in Table 1, the astronomically most interesting lines either have been measured or can now be predicted throughout the entire millimeter domain to better than $50 \mathrm{kHz}$ up to $250 \mathrm{GHz}\left(0.06 \mathrm{~km} \mathrm{~s}^{-1}\right.$ in terms of radial velocity).

\subsection{Poor predictive power of extrapolating high-frequency transitions from microwave measurements}

While frequency predictions based on the spectroscopic parameters from Tanimoto et al. (1979) and Tanimoto \& Macdonald

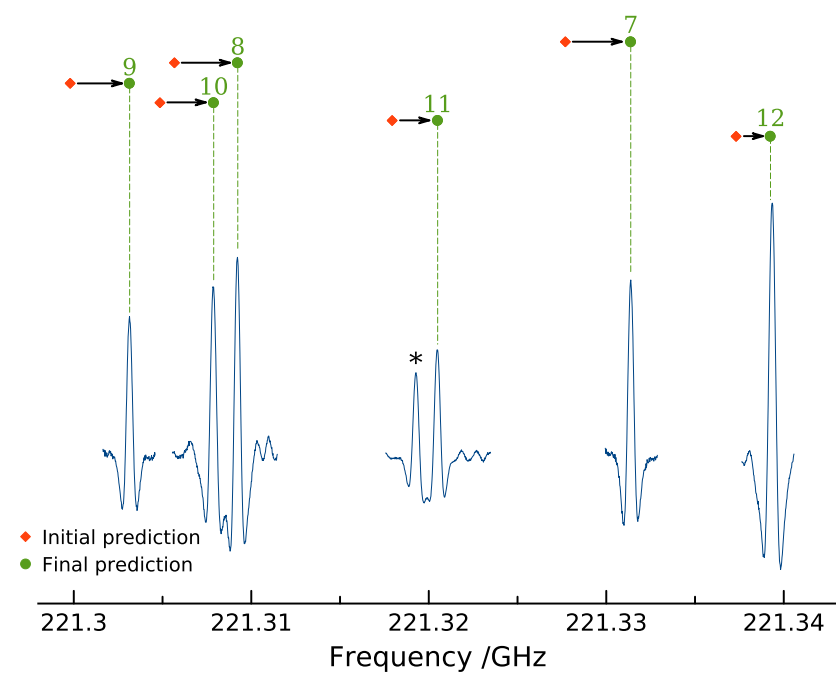

Fig. 5. Individual transitions measured in the ${ }^{q} R(19)$ branch of syn vinyl mercaptan and comparison between the initial (red diamonds) and final (green dots) predictions. The $K_{\mathrm{a}}$ value of each transition is indicated in green; the asymmetry splitting is not resolved for these lines. The transition labeled with an asterisk is unidentified. The line shape appears as a second derivative owing to the employed detection scheme.

(1979) have enabled many new lines to be assigned in the millimeter-wave band, this parameter set alone is inadequate to detect lines of vinyl mercaptan in astronomical sources. Many high- $J$, high- $K_{\mathrm{a}}$ transitions, for example, were shifted from their initial predictions by a few to up to tens of $\mathrm{MHz}$; e.g., the transition $16_{314}-16_{215}$ initially predicted at $226581.2 \mathrm{MHz}$ was measured at $226532.7 \mathrm{MHz}$. In space, the most relevant transitions are the ${ }^{q} R$ series for the syn isomer $\left(\Delta J=1, \Delta K_{\mathrm{a}}=0\right)$ as these are the strongest lines at temperatures ranging from a few tens to a few hundreds of K. For these lines, the typical deviation from the initial prediction was in the range $2-5 \mathrm{MHz}$ around $200 \mathrm{GHz}$ (Fig. 5), corresponding to a significant fraction of the $2-10 \mathrm{~km} \mathrm{~s}^{-1}$ linewidths that typify hot cores and corinos in starforming regions. This example illustrates the pitfalls of extrapolating from the microwave to the millimeter and submillimeter domain, particularly for non-rigid molecules, and emphasizes the value of high-frequency laboratory measurements of astrophysically relevant species, even when microwave spectrum data are already available.

\subsection{Isomers}

The relative stability of isomers may be an important factor in predicting isomeric abundances in the ISM. With this motivation in mind, we performed high-level ab initio calculations (Fig. 2) to determine the relative stability of $\left[\mathrm{C}_{2}, \mathrm{H}_{4}, \mathrm{~S}\right]$ isomers and the corresponding isovalent oxygen analogs; for the former, the calculations presented are - to the knowledge of the authors the first report of the relative energetics at $0 \mathrm{~K}$. Because our calculations for the $\left[\mathrm{C}_{2}, \mathrm{H}_{4}, \mathrm{O}\right]$ isomeric family are in excellent agreement with experiments, we can in turn be confident of the $\left[\mathrm{C}_{2}, \mathrm{H}_{4}, \mathrm{~S}\right]$ thermochemistry, which is significantly less well constrained by measurements.

In contrast to the oxygen isomers, the four lowest single $\left[\mathrm{C}_{2}, \mathrm{H}_{4}, \mathrm{~S}\right]$ isomers are effectively isoenergetic at our current level of theoretical sophistication. While it is unclear if a higher level of treatment (requiring sub-kJ/mol) changes the energetic ordering of these isomers, the near-degeneracy of $\left[\mathrm{C}_{2}, \mathrm{H}_{4}, \mathrm{~S}\right]$ isomers 
Table 2. Parameters of our best-fit LTE model (or upper limit) of selected complex organic molecules toward Sgr B2(N2).

\begin{tabular}{|c|c|c|c|c|c|c|c|c|c|c|}
\hline Molecule & Status $^{a}$ & $N_{\mathrm{det}}^{b}$ & $\begin{array}{l}\mathrm{Size}^{c} \\
\left({ }^{\prime \prime}\right)\end{array}$ & $\begin{array}{c}T_{\text {rot }}{ }^{d} \\
(\mathrm{~K})\end{array}$ & $\begin{array}{c}N^{e} \\
\left(\mathrm{~cm}^{-2}\right)\end{array}$ & $C^{f}$ & $\begin{array}{c}\Delta V^{g} \\
\left(\mathrm{~km} \mathrm{~s}^{-1}\right)\end{array}$ & $\begin{array}{c}V_{\mathrm{off}^{h}}^{h} \\
\left(\mathrm{~km} \mathrm{~s}^{-1}\right)\end{array}$ & $\frac{N_{\mathrm{ref}} i}{N} i$ & Ref. \\
\hline $\mathrm{CH}_{3} \mathrm{SH}, v=0^{\star}$ & $\mathrm{d}$ & 12 & 1.40 & 180 & $3.4(17)$ & 1.00 & 5.4 & -0.5 & 1 & 2 \\
\hline gauche $-\mathrm{C}_{2} \mathrm{H}_{5} \mathrm{SH}$ & $\mathrm{n}$ & 0 & 1.40 & 180 & $<1.6(16)$ & 1.95 & 5.4 & -0.5 & $>22$ & 2 \\
\hline syn- $\mathrm{C}_{2} \mathrm{H}_{3} \mathrm{SH}$ & $\mathrm{n}$ & 0 & 1.40 & 180 & $<3.7$ (16) & 1.22 & 5.4 & -0.5 & $>9$ & 1 \\
\hline anti- $\mathrm{C}_{2} \mathrm{H}_{3} \mathrm{SH}$ & $\mathrm{n}$ & 0 & 1.40 & 180 & $<1.6(16)$ & 1.63 & 5.4 & -0.5 & $>21$ & 1 \\
\hline $\mathrm{CH}_{3} \mathrm{CN}, v_{8}=1^{\star}$ & $\mathrm{d}$ & 20 & 1.40 & 170 & $2.2(18)$ & 1.00 & 5.4 & -0.5 & 1 & 3 \\
\hline $\mathrm{C}_{2} \mathrm{H}_{5} \mathrm{CN}, v=0$ & $\mathrm{~d}$ & 154 & 1.20 & 150 & $6.2(18)$ & 1.38 & 5.0 & -0.8 & 0.35 & 3 \\
\hline $\mathrm{C}_{2} \mathrm{H}_{3} \mathrm{CN}, v=0$ & $\mathrm{~d}$ & 44 & 1.10 & 200 & $4.2(17)$ & 1.00 & 6.0 & -0.6 & 5 & 3 \\
\hline $\mathrm{CH}_{3} \mathrm{OH}, v_{\mathrm{t}}=1^{\star}$ & $\mathrm{d}$ & 16 & 1.40 & 160 & $4.0(19)$ & 1.00 & 5.4 & -0.2 & 1 & 2 \\
\hline $\mathrm{C}_{2} \mathrm{H}_{5} \mathrm{OH}$ & d & 168 & 1.25 & 150 & $2.0(18)$ & 1.24 & 5.4 & 0.0 & 20 & 2 \\
\hline syn- $\mathrm{C}_{2} \mathrm{H}_{3} \mathrm{OH}$ & $\mathrm{n}$ & 0 & 1.25 & 150 & $<4.0(16)$ & 1.00 & 5.4 & 0.0 & $>1000$ & 1 \\
\hline anti- $\mathrm{C}_{2} \mathrm{H}_{3} \mathrm{OH}$ & $\mathrm{n}$ & 0 & 1.25 & 150 & $<1.2(16)$ & 1.00 & 5.4 & 0.0 & $>3333$ & 1 \\
\hline
\end{tabular}

Notes. ${ }^{(a)}$ d: detection, n: non-detection. ${ }^{(b)}$ Number of detected lines (conservative estimate, see Sect. 3 of Belloche et al. 2016). One line of a given species may mean a group of transitions of that species that are blended together. ${ }^{(c)}$ Emission diameter $(F W H M) .{ }^{(d)}$ Rotational temperature. ${ }^{\left({ }^{(e)}\right.}$ Total column density of the molecule, except for the syn and trans conformers of vinyl mercaptan and vinyl alcohol, which are in each case considered as independent species. For these species, the column density represents the column density of each conformer separately. $X(Y)$ means $X \times 10^{Y}$. ${ }^{(f)}$ Correction factor that was applied to the column density to account for the contribution of vibrationally or torsionally excited states or other conformers, in the cases where this contribution was not included in the partition function of the spectroscopic predictions. ${ }^{(g)}$ Linewidth $(F W H M)$. ${ }^{(h)}$ Velocity offset with respect to the assumed systemic velocity of Sgr B2(N2), $V_{\mathrm{lsr}}=74 \mathrm{~km} \mathrm{~s}^{-1}$. ${ }^{(i)}$ Column density ratio, with $N_{\text {ref }}$ the column density of the previous reference species marked with a $\star$.

References. (1) This work, (2) Müller et al. (2016), (3) Belloche et al. (2016).

presents a fairly unique test bed to assess possible reaction pathways in astrochemical objects. The role of icy grains in $\left[\mathrm{C}_{2}, \mathrm{H}_{4}, \mathrm{O}\right]$ formation has been suggested as the underlying factor as to why abundance ratios depart from thermodynamic equilibrium (Bennett et al. 2005). Having established that both isomers of vinyl mercaptan and thiirane are effectively isoenergetic, their relative interstellar abundance should present a more sensitive probe of local processes (e.g., shock and grain chemistry) than their oxygen analogs, which are more widely spaced in energy (Fig. 2). A systematic astronomical investigation of the $\left[\mathrm{C}_{2}, \mathrm{H}_{4}, \mathrm{~S}\right]$ family of isomers, however, is currently limited by the lack of reliable frequencies in the millimeter and submillimeter domains for thioacetaldehyde, which is the remaining isomer for which no data has presently been published beyond the microwave domain.

\subsection{Astronomical searches}

Searches for the syn and anti isomers of vinyl mercaptan were performed with the EMoCA spectrum of Sgr B2(N2) using the best-fit parameters in Table 1. Weeds (Maret et al. 2011) was used to produce synthetic spectra of both isomers under the local thermodynamic equilibrium (LTE) approximation, and the same parameters (emission size, rotational temperature, linewidth, and velocity offset with respect to the assumed systemic velocity of the source) as those derived for methyl mercaptan in Müller et al. (2016) were assumed. Hence, the only free parameters are the column densities of the syn and anti forms of vinyl mercaptan, which we consider as independent species.

No evidence was found for either isomer in the EMoCA spectrum. The upper limits of the column densities are reported in Table 2, along with the column densities or upper limits thereof derived for other molecules in our previous EMoCA studies (Müller et al. 2016; Belloche et al. 2016). In addition, no evidence was found for the syn and anti isomers of vinyl alcohol using predictions derived from the pure rotational works reported in Saito (1976) and Rodler (1985). The corresponding upper limits of their column densities are summarized in Table 2 as well. The non-detection of vinyl alco- hol with ALMA along with its non-detection with the Institut de Radioastronomie Millimétrique (IRAM) $30 \mathrm{~m}$ telescope (Belloche et al. 2013) confirms that if vinyl alcohol were present in the Sgr B2(N) star-forming region at the level reported by Turner \& Apponi (2001), whose observations with the Kitt Peak $12 \mathrm{~m}$ telescope did not resolve the multiple hot cores embedded in Sgr $\mathrm{B} 2(\mathrm{~N})$, then its emission must arise from the source envelope on large scales $(>1 \mathrm{pc})$ and not from the much more compact hot cores, as already stated by Belloche et al. (2013). Surveys like the Prebiotic Interstellar Molecular Survey (PRIMOS, Neill et al. 2012) or the survey performed at the Arizona Radio Observatory (ARO; Halfen et al. 2017) may shed more light on the presence and distribution of vinyl alcohol toward Sgr B2(N).

We find that the syn and anti isomers of vinyl mercaptan are at least 9 and 21 times less abundant than methyl mercaptan, respectively. The energy difference between the syn and anti isomers expressed in temperature units is about $60 \mathrm{~K}$, which implies that the abundance ratio of the anti to syn isomers is 0.72 for a temperature of $180 \mathrm{~K}$. This means that the total column density of vinyl mercaptan at this temperature is 1.72 times that of the syn isomer or equivalently 2.4 times that of the anti isomer. The most stringent constraint on the total column density of vinyl mercaptan is provided by the anti isomer: vinyl mercaptan is at least $\sim 9$ times (21/2.4) less abundant than methyl mercaptan.

The lower limit for the abundance ratio of vinyl to methyl mercaptan can be compared to ratios obtained for other families of molecules. It is nearly a factor of two higher than the abundance ratio measured for the cyanides $\left(\left[\mathrm{CH}_{3} \mathrm{CN}\right] /\left[\mathrm{C}_{2} \mathrm{H}_{3} \mathrm{CN}\right]=5\right)$. For vinyl alcohol, the large energy difference between the syn and anti isomers ( $4.6 \mathrm{~kJ} \mathrm{~mol}^{-1}$, i.e., $385 \mathrm{~cm}^{-1}$, Fig. 2) implies that the total column density of vinyl alcohol is equal to 1.025 times that of the syn isomer at $150 \mathrm{~K}$. We thus conclude that vinyl alcohol is at least 1000 times less abundant than methanol. This lower limit is more than two orders of magnitude higher than the ratio measured for the cyanides. Given that the abundance ratio of methanol to ethanol is also higher (by a factor $\sim 60$ ) than the abundance ratio of the corresponding cyanides, the chemistries of the cyanides and alcohols appear to differ significantly. However, the observational 
constraints do not tell us whether the chemistries of the mercaptans and alcohols are similar or differ: the lower limit to the ratio of methyl to ethyl mercaptan is marginally consistent with the corresponding ratio measured for the alcohols, and the lower limits to the ratio of the methyl to vinyl species do not exclude a similar ratio in both families.

\section{Conclusions}

In the present study, a rotational investigation of the two isomers of vinyl mercaptan has been extended up to $250 \mathrm{GHz}$. The new set of transition frequencies and derived spectroscopic constants provide a highly reliable basis for subsequent searches for both species in the ISM. Calculations of the rotational spectra are available in the catalog section of the CDMS (Endres et al. 2016) database $^{2}$ while linelists, parameters, fits, and auxiliary files are available in the data section ${ }^{3}$.

The work performed in this study highlights the urgent need for high-frequency measurements of numerous species of interstellar interest that so far have only been characterized at centimeter wavelengths. Indeed, most of the current ISM observations are now performed in the millimeter and submillimeter domains, using facilities such as ALMA, NOEMA, APEX, and SOFIA, but frequency extrapolations into the millimeter domain using spectroscopic constants derived from low $J$ and $K_{\mathrm{a}}$ observations are inherently uncertain, and make detection of new astronomical species unduly challenging. Additionally, this work provides constraints on isomeric populations in the ISM. Both syn and anti vinyl mercaptan have been the subjects of searches in the EMoCA survey recorded toward the hot core Sgr B2(N2). No transitions of either species has been detected and upper limits for their column densities have been derived, revealing that vinyl mercaptan is at least nine times less abundant than methyl mercaptan in this source.

Acknowledgements. Part of this work is carried out within the Collaborative Research Centre 956, sub-project B3, funded by the Deutsche Forschungsgemeinschaft (DFG) - project ID 184018867, and the NSF grant AST-1615847. M.-A. M.-D. is thankful to the Programme National "Physique et Chimie du Milieu Interstellaire" (PCMI) of CNRS/INSU with INC/INP co-funded by CEA and CNES for support. M.C.M. thanks NASA grants NNX13AE59G and 80NSSC18K0396.

\section{References}

Almond, V., Charles, S. W., MacDonald, J. N., \& Owen, N. L. 1977, J. Chem. Soc. Chem. Commun., 483

Almond, V., Charles, S. W., MacDonald, J. N., \& Owen, N. L. 1983, J. Mol Struct., 100, 223

Almond, V., Permanand, R., \& Macdonald, J. 1985, J. Mol. Struct., 128, 337

Anderson, D. E., Bergin, E. A., Maret, S., \& Wakelam, V. 2013, ApJ, 779, 141

Bane, M. K., Thompson, C. D., Appadoo, D. R. T., \& McNaughton, D. 2012, J. Chem. Phys., 137, 084306

Belloche, A., Müller, H. S. P., Menten, K. M., Schilke, P., \& Comito, C. 2013, A\&A, 559, A47

Belloche, A., Müller, H. S. P., Garrod, R. T., \& Menten, K. M. 2016, A\&A, 587 A91

Bennett, C. J., Osamura, Y., Lebar, M. D., \& Kaiser, R. I. 2005, ApJ, 634, 698

Bockelee-Morvan, D., Lis, D. C., Wink, J. E., et al. 2000, A\&A, 353, 1101

Bomble, Y. J., Vazquez, J., Kallay, M., et al. 2006, J. Chem. Phys., 125, 064108, wOS:000239765100010

Boogert, A. C. A., Schutte, W. A., Helmich, F. P., Tielens, A. G. G. M., \& Wooden, D. H. 1997, A\&A, 317, 929
Bunn, H., Hudson, R. J., Gentleman, A. S., \& Raston, P. L. 2017, ACS Earth Space Chem., 1, 70

Cazzoli, G., Lattanzi, V., Kirsch, T., et al. 2016, A\&A, 591, A126

Chin, W., Mok, C., \& Huang, H. 1994, J. Electron. Spectrosc. Relat. Phenom., 67, 173

Coriani, S., Marcheson, D., Gauss, J., et al. 2005, J. Chem. Phys., 123, 184107

Cunningham, Jr., G. L., Boyd, A. W., Myers, R. J., Gwinn, W. D., \& Le Van, W. I. 1951, J. Chem. Phys., 19, 676

Dickens, J. E., Irvine, W. M., Ohishi, M., et al. 1997, ApJ, 489, 753

Dunning, T. H., Peterson, K. A., \& Wilson, A. K. 2001, J. Chem. Phys., 114, 9244

Endres, C. P., Schlemmer, S., Schilke, P., Stutzki, J., \& Muller, H. S. P. 2016, J. Mol. Spectrosc., 327, 95

Gottlieb, C. A. 1973, in Molecules in the Galactic Environment, eds. M. A. Gordon, \& L. E. Snyder, 181

Halfen, D. T., Woolf, N. J., \& Ziurys, L. M. 2017, ApJ, 845, 158

Harding, M. E., Metzroth, T., Gauss, J., \& Auer, A. A. 2008a, J. Chem. Theory Comput., 4, 64

Harding, M. E., Vazquez, J., \& Ruscic, B. 2008b, J. Chem. Phys., 128, 114111

Hirao, T., Okabayashi, T., \& Tanimoto, M. 2001, J. Mol. Spectr., 208, 148

Jimenez-Escobar, A., \& Munoz Caro, G. M. 2011, A\&A, 536, A91

Joseph, C. L., Snow, T. P., Seab, C. G., \& Crutcher, R. M. 1986, ApJ, 309, 771

Karton, A., \& Talbi, D. 2014, Chem. Phys., 436-437, 22

Kolesniková, L., Tercero, B., Cernicharo, J., et al. 2014, ApJ, 784, L7

Kroto, H. W., Landsberg, B. M., Suffolk, R. J., \& Vodden, A. 1974, Chem. Phys. Lett., 29, 265

Lattelais, M., Pauzat, F., Ellinger, Y., \& Ceccarelli, C. 2009, Astrophys. Lett., 696, L133

Loomis, R. A., McGuire, B. A., Shingledecker, C., et al. 2015, ApJ, 799, 34

Maret, S., Hily-Blant, P., Pety, J., Bardeau, S., \& Reynier, E. 2011, A\&A, 526, A47

Martin-Drumel, M., van Wijngaarden, J., Zingsheim, O., et al. 2015, J. Mol. Spectr., 307, 33

Martin-Domenech, R., Jimenez-Serra, I., Munoz Caro, G. M., et al. 2016, A\&A, 585, A112

McCarthy, M. C., Chen, W., Travers, M. J., \& Thaddeus, P. 2000, ApJS, 129, 611

Menten, K. M., Wyrowski, F., Belloche, A., et al. 2011, A\&A, 525, A77

Müller, H. S. P., Belloche, A., Xu, L.-H., et al. 2016, A\&A, 587, A92

Neill, J. L., Muckle, M. T., Zaleski, D. P., et al. 2012, ApJ, 755, 153

Neufeld, D. A., Falgarone, E., Gerin, M., et al. 2012, A\&A, 542, L6

Neufeld, D. A., Godard, B., Gerin, M., et al. 2015, A\&A, 577, A49

Nobes, R. H., Radom, L., \& Allinger, N. L. 1981, J. Mol. Struct., 85, 185

Palumbo, M. E., Tielens, A. G. G. M., \& Tokunaga, A. T. 1995, ApJ

Peterson, K. A., \& Dunning, T. H. 2002, J. Chem. Phys., 117, 10548

Pickett, H. M. 1991, J. Mol. Spectr., 148, 371

Pickett, H. M., Poynter, R. L., Cohen, E. A., et al. 1998, J. Quant. Spectr. Rad. Transfer, 60, 883

Plant, C., Boggs, J., Macdonald, J., \& Williams, G. 1992, Struct. Chem., 3, 3

Raghavachari, K., Trucks, G. W., Pople, J. A., \& Head-Gordon, M. 1989, Chem. Phys. Lett., 157, 479

Rodler, M. 1985, J. Mol. Spectr., 114, 23

Saito, S. 1976, Chem. Phys. Lett., 42, 399

Savage, B. D., \& Sembach, K. R. 1996, ARA\&A, 34, 279

Scappini, F., Cecchi-Pestellini, C., Smith, H., Klemperer, W., \& Dalgarno, A. 2003, MNRAS, 341, 657

Smith, R. G. 1991, MNRAS, 249, 172

Stanton, J. F., Gauss, J., Harding, M. E., \& Szalay, P. G. 2017, cFour, a quantum chemical program package for the current version, see http://www . cfour . de

Tanimoto, M., \& Macdonald, J. 1979, J. Mol. Spectr., 78, 106

Tanimoto, M., \& Saito, S. 1977, Chem. Lett., 6, 637

Tanimoto, M., Almond, V., Charles, S., Macdonald, J., \& Owen, N. 1979, J. Mol. Spectr., 78, 95

Thorwirth, S., \& Harding, M. E. 2009, J. Chem. Phys., 130, 214303

Thorwirth, S., Kaiser, R. I., Crabtree, K. N., \& McCarthy, M. C. 2015, Chem. Commun., 51, 11305

Tieftrunk, A., Forets, G. P. D., Schilke, P., \& Walmsley, C. M. 1994, A\&A, 289, 579

Turner, B. E., \& Apponi, A. J. 2001, ApJ, 561, L207

Wakelam, V., Caselli, R., Ceccarelli, C., Herbst, E., \& Castets, A. 2004, A\&A, 422, 159

\footnotetext{
2 https://cdms.astro.uni-koeln.de/classic/entries/

3 https://cdms.astro.uni-koeln.de/classic/

predictions/daten/VySH/
} 
Appendix A: Molecular structures of the $\left[\mathrm{C}_{2}, \mathrm{H}_{4}, \mathrm{~S}\right]$ isomers

Internal coordinates ae-CCSD (T)/cc-pwCVQZ

syn vinyl mercaptan

$\mathrm{H}$

C 1 r 1

C 2 r2 1 a1

S 3 r3 2 a2 1 dQ

$\mathrm{H} \quad 4$ r4 3 a3 2 dQ

H 2 r5 3 a 4 1 1 d 180

H 3 r6 2 a5 6 d0

$\begin{array}{llr}\mathrm{r} 1 & = & 1.08115 \\ \mathrm{r} 2 & = & 1.33270 \\ \mathrm{a} 1 & = & 122.05669 \\ \mathrm{r} 3 & = & 1.75221 \\ \mathrm{a} 2 & = & 127.33692 \\ \mathrm{~d} 0= & 0.00000 \\ \mathrm{r} 4= & 1.33663 \\ \mathrm{a} 3 & = & 96.56932 \\ \mathrm{r} 5 & = & 1.07962 \\ \mathrm{a} 4 & = & 120.07569 \\ \mathrm{~d} 180= & 180.00000 \\ \mathrm{r} 6 & = & 1.08206 \\ \mathrm{a} 5 & = & 120.75551\end{array}$

anti vinyl mercaptan

$\mathrm{H}$

C 1 r 1

C 2 r2 1 a1

S 3 r3 32 a2 1 d1

H 4 r4 3 a3 2 d2

$\begin{array}{llllllll}\mathrm{H} & 2 & \mathrm{r} 5 & 3 & \mathrm{a} 4 & 1 & \mathrm{~d} 3\end{array}$

H 3 r6 2 a5 6 d4

$\begin{array}{rlr}\mathrm{r} 1 & = & \\ \mathrm{r} 2 & = & 1.08105 \\ \mathrm{a} 1 & = & 12.33250 \\ \mathrm{r} 3 & = & 1.79666 \\ \mathrm{a} 2 & = & 122.52864 \\ \mathrm{~d} 1 & = & 3.65449 \\ \mathrm{r} 4 & = & 1.33431 \\ \mathrm{a} 3 & = & 96.59150 \\ \mathrm{~d} 2 & = & -159.42502 \\ \mathrm{r} 5 & = & 1.07959 \\ \mathrm{a} 4 & = & 120.21684 \\ \mathrm{~d} 3 & = & -179.52134\end{array}$

$\begin{array}{rrr}\mathrm{r} 6 & = & 1.08069 \\ \mathrm{a} 5 & = & 121.24236 \\ \mathrm{~d} 4 & = & 0.62115\end{array}$

thiirane

$\mathrm{S}$

C 1 r 1

C $\begin{array}{lllll} & \mathrm{r} 1 & 1 & \mathrm{a} 1\end{array}$

H 2 r2 1 a2 3 d1

H 2 r2 1 a2 3 md1

$\mathrm{H} \quad 3$ r2 1 a2 2 d1

H 3 r2 1 a2 2 md1

$\begin{array}{llr}\mathrm{r} 1 & = & 1.81067 \\ \mathrm{a} 1= & 48.27245 \\ \mathrm{r} 2= & 1.07991 \\ \mathrm{a} 2= & 115.09725 \\ \mathrm{~d} 1= & 110.93875 \\ \mathrm{~m} 1= & -110.93875\end{array}$

thioacetaldehyde

$\mathrm{S}$

C 1 r 1

C $\begin{array}{llll}2 & \text { r2 } & 1 & \text { a1 }\end{array}$

H 3 r3 2 a2 1 do

H $2 \begin{array}{rlllll}\text { r } 4 & 3 & \text { a3 } & 1 & \text { d } 180\end{array}$

H 3 r5 2 a4 5 d1

H 3 r5 2 a4 5 md1

$\begin{array}{llr}\mathrm{r} 1 & = & 1.61419 \\ \mathrm{r} 2 & = & 1.49172 \\ \mathrm{a} 1= & 125.71288 \\ \mathrm{r} 3= & 1.08597 \\ \mathrm{a} 2= & 111.24050 \\ \mathrm{~d} 0= & 0.00000 \\ \mathrm{r} 4= & 1.08887 \\ \mathrm{a} 3= & 115.25385 \\ \mathrm{~d} 180= & 180.00000 \\ \mathrm{r} 5= & 1.09181 \\ \mathrm{a} 4= & 109.69777 \\ \mathrm{~d} 1= & 58.47364 \\ \mathrm{~m} 11= & -58.47364\end{array}$

Appendix B: Observed experimental transitions of vinyl mercaptan

Tables B.1 and B.2 are available at the CDS. 